\title{
Ejemplo de proyecto docente innovador: caso Comunica2
}

DOI: $10.46932 / \mathrm{sfjdv} 1 \mathrm{n} 4-010$

Received in: August 1st, 2020

Accepted in: September 30th, 2020

\author{
Marga Cabrera \\ Higher academic background: Phd in Audiovisual Communication. Professor \\ Current Institution: Universitat Politécnica de Valencia \\ Full address: Camí de Vera, s/n, 46022 València Spain \\ E-mail: mcabrera@upv.es \\ Rebeca Díez-Somavilla \\ Higher academic background: Phd in Audiovisual Communication. Professor \\ Current Institution: Universitat Politécnica de Valencia \\ Full address: Camí de Vera, s/n, 46022 València Spain \\ E-mail: rdiez@har.upv.es
}

\begin{abstract}
Alberto Sancho
Higher academic background: Researcher

Current Institution: Universitat Politécnica de Valencia

Full address: Camí de Vera, s/n, 46022 València Spain

E-mail: sgalberto@gmail.com
\end{abstract}

\begin{abstract}
El congreso internacional Comunica2 sobre redes sociales es un proyecto docente en el que se involucran hasta 60 alumnos de comunicación audiovisual, turismo y ex graduados. La idea principal es que sean ellos mismos los que lideren, programen y ejecuten todo el trabajo de comunicación y difusión del evento, así como la gestión del mismo. La novedad estriba en que es una formación totalmente transversal dado que son los mismos alumnos, apoyados por los técnicos, docentes, ex alumnos y empresas colaboradoras, los que forman a las nuevas generaciones. Los contenidos también son transversales pues aprenden de gestión de eventos, difusión en redes, producción y postproducción, relación con la prensa y de relaciones públicas. Así los antiguos alumnos que están ya en el ámbito laboral (y pueden) regresan unos días a las aulas para trabajar y formar a los más jóvenes que empiezan. La experiencia de siete ediciones nos ha permitido tener un flujo continuo de equipo capaz de formar a nuevos estudiantes. Los resultados son espectaculares pues cada año son más los que se adhieren a la experiencia, todos repiten y están entusiasmados con formar a nuevos en la materia.
\end{abstract}

Keywords: formación transversal, innovación, proyecto docente, innovación universitária.

\section{INTRODUCCIÓN}

El congreso internacional Comunica2 sobre redes sociales es en realidad un proyecto docente en el que se involucran hasta 60 alumnos de comunicación audiovisual. La ideal principal es que sean ellos mismos los que hagan todo el trabajo de comunicación y difusión del evento, así como la gestión del mismo. Entendemos que es una nueva forma en cuanto a enseñanza e investigación del periodismo y la 
comunicación: un planteamiento innovador por su formato transversal, en cuanto a contenidos pues se van aprendiendo sobre la marcha, y en cuanto a docentes y alumnado y sus relaciones totalmente trasversales. Es fundamental formar a los estudiantes de manera integral para fomentar un conjunto de competencias transversales a través de metodologías activas que impliquen una mayor participación del estudiante (Alias et. Al. 2006).

Los que lideran el proyecto son dos profesoras, el técnico de audiovisual del Campus, la técnica de prensa y cinco ex alumnos. Lo más destacable es que los que ya han pasado por esta experiencia van adquiriendo el nivel suficiente para formar a las nuevas generaciones que van viniendo. Así los antiguos alumnos que están ya en el terreno laboral (y pueden) regresan unos días a las aulas para trabajar y formar a los más jóvenes que empiezan. La experiencia de siete ediciones nos ha permitido tener un flujo continuo de equipo capaz de formar a los nuevos. Según diferentes estudios (Johnson 1989 y Slavin 1987), la cooperación ofrece altos niveles de logro, una mayor retención de lo que se ha aprendido, mayor razonamiento y voluntad para desarrollar tareas difíciles y conseguir superarlas; es un aprendizaje cooperativo.

La colaboración con el proyecto es completamente voluntaria, no se les gratifica ni con notas, ni con créditos, ni con dinero. El único beneficio que obtienen es la experiencia a través de un gran trabajo y la inscripción gratuita (insignificante pues para los alumnos es de $35 €$ ).

Cada voluntario puede elegir en qué quiere colaborar y tener experiencia dentro del congreso. Se ofrecen tareas por equipos en: grafismo, retransmisión en directo a través de redes sociales (cuenta de Twitter con el objetivo de llegar al trending topic, generación y mantenimiento de contenidos para Facebook, entradas de blog, resúmenes en Storify, cuenta de Instagram y Snapchat, fotografía...), equipo de streaming (realizadores, ayudantes de realización, responsables de audio-emisión-grafismo, cámaras), regidor de sala, responsables de talleres, responsables de dietas y alojamiento, padrinos y madrinas de ponentes, equipo científico (gestionan las comunicaciones y las aulas donde se exponen, tienen que hacer resumen de cada una de ellas y compartirla con los equipos de redes), relaciones públicas nocturno, gabinete de prensa (mantenimiento de base de datos de medios; notas de prensa previas, de cada ponencia, y al finalizar; recepción de medios y gestión de entrevistas con ponentes, análisis del impacto en medios); y más funciones que se van añadiendo según el número de alumnos voluntarios aumenta.

El congreso ha conseguido unir diferentes generaciones de alumnos en un proyecto en común, les dota de currículo y experiencia y les abre puertas en el sector laboral pues las empresas llaman para solicitar alumnos que hayan participado en el Comunica2. 
Desde que finaliza una edición, todo el equipo se siente involucrado pensando a quién podría invitar como ponente, qué mesas o talleres montar, qué mejorar de otras ediciones, analizan otros congresos para ver qué novedades introducir, se buscan subvenciones o acuerdos con entidades, se "fichan" alumnos de primeros cursos con potencial, y en todo momento se busca innovar.

\section{JUSTIFICACIÓN}

La idea surgió en el 2010 debido a la falta de conocimiento en la materia de los propios profesores de Comunicación Audiovisual. No era lógico que estuviera en boga algo tan patente en el mundo de la comunicación como son las redes sociales y los docentes no tuvieran conocimientos en este aspecto, y mucho menos experiencia. La velocidad a la que irrumpían en ese momento en todos los aspectos de la vida cotidiana no dejaba indiferente, y fueron los propios alumnos de aquel entonces los que propusieron organizar un curso en el que se invitase a algún profesional con experiencia en comunicación a través de redes sociales, por aquel entonces Facebook y Twitter. En el primer planteamiento de curso ya quedó patente la necesidad de ampliar el espectro de profesionales y temas. No era suficiente con montar un curso; era necesario crear una jornada o congreso para poder invitar a diferentes especialistas y aprender, aceleradamente, los cambios que estaban surgiendo en el mundo de la comunicación digital. Cualquier alumno que quisiera entrar en el mundo laboral debía conocer qué se estaba haciendo en las redes sociales, cómo plantear una estrategia, cómo medir los resultados y cómo implementar nuevas narrativas.

Así pues, surgió la idea de montar un congreso mediante el cual aprender tanto docentes como alumnos y empresarios de la zona. Al ser una idea nacida de los alumnos, fueron ellos mismos los que investigaron a quién invitar y qué formato querían darle al evento. Pensaron que además de charlas o ponencias magistrales era necesario pisar tierra y probar ellos mismos la mejor manera de comunicar a través de las redes sociales. Para ello se planificaron talleres prácticos durante el congreso y mesas redondas mediante las cuales poder plantear diferentes formas de entender la comunicación digital. Este trabajo suponía introducir algunas de las innovaciones educativas destacadas por la Open University en su informe sobre Innovating Pedagogy (2014): combinar el aprendizaje dentro y fuera del aula (flipped classroom), aprender a aprender con un aprendizaje efectivo y continuo, aprendizaje basado en eventos, desarrollo de conceptos umbral con ideas y temas problemáticos reales que abren la puerta a nuevas maneras de pensar, y bricolaje o aprender practicando con materiales y recursos de una manera creativa.

A la hora de cubrir los gastos del congreso surgió la necesidad de llegar al máximo posible de asistentes, y así fue como nació también la creación de una web y una estrategia en redes para captar al mayor número posible de interesados, y de esta forma, poder cubrir todas las facturas que iban a derivar 
de traer ponentes de toda España y que no subiese el coste para los alumnos del Campus. Esto obligó a relacionarse con empresarios de la zona como posibles patrocinadores del Congreso, lo que más tarde derivó en que algunos de ellos se involucraran al $100 \%$ en varias tareas.

El reto de gestionar el acceso a ponentes de primer nivel, conseguir financiación, el hecho de tramitar los viajes, resolver el alojamiento, dietas y cuidado de los invitados, hizo que todos ganaran en experiencia en organización de eventos. No se podía dejar la oportunidad de dotarlo de parte científica, y de esta forma conocer las experiencias de investigadores que, a lo largo de toda España, estaban investigando en temas relacionados con las redes sociales, así surgió la parte científica del Comunica2, también de la mano de varios alumnos que estaban ya realizando el doctorado.

Creemos que el proyecto Comunica2 es un proyecto docente transversal e innovador, en tanto que surgió por una necesidad de los alumnos, y que año tras año, las nuevas generaciones han ido aceptando el reto y tomando el relevo, mejorando el formato y formando una gran familia de alumnos y ex alumnos con el único fin de seguir aprendiendo. Es una preparación para el mundo profesional: "Si tienen algún sentido todos los aprendizajes que hacen los alumnos, es porque los preparan para su incorporación a la sociedad en el sentido más amplio del término” (Domènech y Guerrero 2005, p. 22).

\section{OBJETIVOS}

Si bien el proyecto tiene diversos objetivos, vamos a enumerar el que nos mueve a todos, seguido por los objetivos secundarios que año tras año van aumentando en ambición.

\subsection{OBJETIVO PRINCIPAL}

Conseguir conocimiento y aprendizaje en redes sociales y gestión de eventos a través de una experiencia colaborativa en la que se implementan las competencias transversales.

\subsection{OBJETIVOS SECUNDARIOS}

Entre los objetivos secundarios cabe destacar:

Trabajo en equipo a través de diferentes perfiles y niveles de experiencia.

Ambiente sano y distendido en el que no haya jerarquías y todos aprendan: colaborativo.

Transmisión de conocimiento de unas generaciones a otras.

Aprendizaje global, en el que no se tiene en cuenta la nota, sino que es totalmente voluntario.

Acercamiento de investigadores a nivel internacional con aportes interesantes.

Lograr el mayor número de inscripciones posibles hasta llegar al tope del Aula Magna. 
Conseguir ser trending topic lo antes posible para obtener la mayor difusión en redes.

Generación de seguimiento internacional a través del streaming y preguntas vía Twitter.

\section{HIPÓTESIS}

La hipótesis que defendemos y sobre la que se basa la experiencia docente innovadora es: "La formación práctica y transversal no evaluable es posible con trabajo en equipo colaborativo a través de distintas generaciones de alumnos, incluidos los que ya han finalizado los estudios."

\section{METODOLOGÍA}

El congreso internacional Comunica2 sobre redes sociales que se celebra desde el 2010 en el campus de Gandia es, en realidad, un proyecto docente en el que se involucran más de 60 alumnos de Comunicación Audiovisual y algunos de Turismo o Master. La ideal principal es que sean ellos mismos los que hagan el trabajo de comunicación, producción y difusión del evento. Para ello la metodología que empleamos es dividir a todos los alumnos en varios grupos de trabajo, en los que siempre hay, al menos, un involucrado con experiencia en el tema de anteriores ocasiones. Se valora la experiencia en el congreso o en el trabajo asignado, independientemente del curso en el que esté, de esta forma es posible que un alumno de segundo coordine a alumnos ya graduados o en los últimos cursos. Es una educación basada, en gran parte, en la empatía, dado que hoy la neurociencia confirma que sin motivación no hay aprendizaje (Furth 1992), con el objetivo de con conseguir activar el cerebro emocional, porque la educación ha de tender a integrar, conciliar e interrelacionar emoción y razón (Ferrés 2008, p. 60-65)

Los grupos implicados se pueden dividir en:

\subsection{DIRECCIÓN}

El equipo directivo está formado por dos profesoras, junto a un antiguo alumno del campus, este papel ha variado a lo largo de los años según la disponibilidad y experiencia de los antiguos alumnos. Los tres ayudan a trabajar en equipo, enseñan a delegar, muestran el gusto por el trabajo bien hecho y la pasión por un proyecto en común. El profesor se convierte en un aprendiz (Piscitelli 2008) para centrar la educación en logros del mundo real y no en ejemplos (Prensky 2014). Este equipo directivo es el que se encargan de "atraer" al resto de estudiantes para que se comprometan y lo den todo "por amor al arte". También son los que confeccionan el programa y se encargan de convencer a los ponentes para que vengan hasta Gandia sin más pago que las dietas y el viaje. Aceptan sugerencias de todo el resto del equipo para propuestas de mejora o ponentes. 


\subsection{PRODUCCIÓN}

El técnico de audiovisuales del Campus es el encargado de la formación en todo lo relacionado con la grabación y la retransmisión en directo del evento. A su cargo está el equipo de realización: realizadores, ayudantes de realización, responsables de audio-emisión-grafismo, cámaras, regidor de sala... teniendo que organizarse para que la retransmisión sea perfecta durante los dos días que dura el evento.

La técnica del centro de formación de postgrado se encarga de las inscripciones y de los certificados. Forma en la recepción de los asistentes, la preparación de las bolsas de bienvenida y en general en la parte burocrática. Normalmente le ayudan unos cinco alumnos tanto de comunicación audiovisual como de turismo. Se crea una sinergia entre diferentes perfiles de diferentes carreras que consiguen un aprendizaje de diferentes disciplinas casi sin notarlo.

El grafismo de cada edición corre a cargo de una sola persona. Ha habido años que lo ha hecho un alumno y otras un ex alumno. Las tareas que asume son, manteniendo la esencia del logotipo y los colores corporativos, desarrollo del cartel, las camisetas, los banners, las plantillas de power point, el diseño del programa... ¡todo lo que lleve diseño pasa por sus manos! Es mucho trabajo, pero lo asumen con gusto pues es un punto a su favor a la hora de buscar trabajo y demostrar experiencia. Muchos años cuenta con ayuda de alumnos de primeros cursos con menos experiencia y los dirige.

La retransmisión en directo a través de redes sociales la dirige un antiguo alumno y coordinada a un equipo de cinco personas, alumnos y ex alumnos que se encargan de llevar la cuenta de Twitter con el objetivo de llegar a ser trending topic nacional en el menor tiempo posible (ya es un clásico), llevan la generación y mantenimiento de contenidos para Facebook, entradas del blog (a lo largo del año y los días del evento), resúmenes en Storify, gestionan la cuenta de Instagram y Snapchat, generan material audiovisual para compartir: videos, fotografías,... y sobre todo responden rápidamente a cualquier pregunta que venga por redes.

Otro grupo de producción son los fotógrafos, formado por unos tres alumnos y dirigidos generalmente por un ex alumno que haya asumido esta tarea en el pasado. Hacen fotografías del evento, del ambiente y una sesión de retrato a cada uno de los ponentes y compañeros que pasa por el congreso, para después regalarle las fotos y que de alguna manera se lleven un bonito recuerdo de calidad.

Existe un equipo denominado "padrinos" que se encargan de las relaciones públicas del congreso. Cada ponente tiene asignado un padrino que le cuida desde que sale de casa hasta que vuelve. Está a su disposición para cualquier duda o necesidad que pueda surgir. Realmente son los ponentes los que acaban apadrinando al alumno, pues la relación que se genera entre los dos hace que exista la 
confianza suficiente para que más adelante el alumno le pueda pedir ayuda o contactos en el mundo laboral.

De la intendencia y alojamiento se encarga otro grupo y tienen que negociar con los hoteles y el ayuntamiento para conseguir el mejor sitio en la playa de Gandia, además de las mejores vistas. Reservan en restaurantes, bares y todo lo necesario para recibir a los ponentes y asistentes de la mejor manera. No podemos pagar alojamiento y dietas a los asistentes, pero sí podemos negociar precios amigos en distintos lugares. Queremos que todo el mundo se vaya contento.

\subsection{PUBLICIDAD}

Todos los años se monta un equipo de alumnos encargado del anuncio publicitario, "el spot”. En este caso se elige un director entre los alumnos y él mismo se encarga de buscar el equipo y coordinar todo, no tienen a nadie que les marque condiciones, se les deja libertad total. Este año el director ha sido un alumno de tercero y contado con la participación de 20 alumnos, asumiendo las tareas de: dirección, guion, dirección de fotografía, foto fija, storyboard, dirección de arte, dirección de sonido, música, diseño gráfico, producción, montaje, etalonaje, efectos y posproducción, script...

El equipo de redes sociales, dirigido por un antiguo alumno con experiencia en estrategia en redes, es el encargado de viralizar el spot, así como de gestionar la publicidad en Facebook y Google, y de generar la conversación previa al evento para que llegue a la mayor cantidad de usuarios posible a nivel internacional.

\subsection{PRENSA Y RELACIONES CON LOS MEDIOS}

La técnica responsable de prensa del Campus, junto con una de las directoras que es periodista, forma a los alumnos en la redacción de notas de prensa, llevan el mantenimiento de base de datos de medios; hacen las notas de prensa previas, de cada ponencia, y al finalizar; coordinan la recepción de medios y gestión de entrevistas con ponentes, análisis del impacto en medios. Al finalizar el evento recopilan todo el material y hacen un dosier de prensa de cada edición.

\subsection{COMITÉ CIENTÍFICO}

La tarea de coordinador del comité científico suele ir al cargo de un ex alumno que en años anteriores haya ayudado en estas tareas. Se encarga de localizar y gestionar al comité científico, recepcionar las comunicaciones y distribuirlas de forma que se revisen por pares y sean totalmente anónimas. Una vez seleccionadas coordina un equipo de alumnos para gestionar las comunicaciones y las aulas donde se exponen, tienen que hacer resumen de cada una de ellas y compartirla con los equipos 
de redes. También coordina a profesores del campus que nos ayudan con la presentación y moderación de las comunicaciones.

\subsection{POST-PRODUCCIÓN}

Cada edición conlleva un arduo trabajo una vez finalizado el evento. Todo el material recopilado en video y fotografía se edita y postproduce para subirlo a Youtube y generar así un repositorio con lo que ha acontecido en cada una de las ediciones.

\section{RESULTADOS}

Se ha conseguido que el congreso llegue a la categoría internacional, pues los alumnos han sido capaces de lograr que haya financiación suficiente para traer a un experto de otro país. Han sido tres las ediciones internacionales, en las que se ha contado con Esther Vargas (Perú), Alejandro Piscitelli (Argentina) y Janine Warner (Estados Unidos).

También se ha conseguido aumentar el número de asistentes, llegando a 250 inscritos en la edición del 2017 y 2.000 usuarios a través del streaming. En las redes sociales se ha aumentado en cifra de seguidores, teniendo en Twitter: 5.000, Facebook: 2.000, Instagram: 330 y Youtube: 336.

Que el congreso sea en Gandia es un hándicap a la hora de que vengan desde otras ciudades, pues no está bien comunicada como puede estarlo Valencia, sin embargo, los asistentes valoran la situación privilegiada cerca de la playa, el ambiente familiar del campus y la cercanía y complicidad que se crea entre público y ponentes. Además, el Ayuntamiento de Gandia apoya el congreso desde el inicio y es consciente de la repercusión que tiene para la ciudad. En el 2007 ha galardonado al campus de Gandia por su congreso Comunica2 en la Gala de Premios de Turismo por la promoción que se hace de la ciudad.

\section{CONCLUSIONES}

Partiendo de la hipótesis inicial que era si es posible "la formación práctica y transversal no evaluable con trabajo en equipo colaborativo a través de distintas generaciones de alumnos, incluidos los que ya han finalizado los estudios.” Podemos decir rotundamente que sí es posible. Ahora bien, es necesario una gran implicación por parte de los docentes y técnicos involucrados para que todo el equipo se sienta parte de un mismo proyecto y den lo máximo de sí mismos.

Se trata de trabajar soft skills, valores que no se suelen trabajar en los grados universitarios y que cada día son más necesarios y tenidos en cuenta por el mercado laboral. Valores como el trabajo en equipo, la delegación y supervisión de tareas, la responsabilidad frente a un trabajo que depende de cada 
uno, la pérdida de la inocencia, o más bien el despertar de todo lo que es capaz cada uno, y en definitiva las habilidades humanas y de compañerismo, donde cada uno hace y ofrece lo que mejor se le da o para lo que quiere especializarse en el fututo.

Efectivamente, tras siete ediciones y con la experiencia de varias generaciones de alumnos se puede considerar que el Comunica2 es un proyecto docente innovador, transversal y que contribuye al desarrollo de las competencias educativas.

\section{BIBLIOGRAFÍA}

Alías A., Gil, C., Riscos, A., Valcárcel M., Vicario E. (eds). (2006). Acción tutorial a través del portafolios digital en un entorno de aprendizaje cooperativo. En Actas del Encuentro sobre la Formación del Profesorado Universitario. Universidad de Almería. 2006. Recuperado de: http://pareto.ual.es/cgil/documents/portfolioConso.pdf

Domènech, J. Guerrero, J. (2005). Miradas a la educación que queremos. Barcelona: Graó.

Carr N. (2011). Superficiales ¿Qué está haciendo internet con nuestras mentes?.- Madrid: Ed. Taurus.

Castaño, C.; Maíz, I.; Palacio, G. \& Villarroel J. D. Prácticas educativas en entornos Web 2.0. Síntesis. Madrid.(2008)

Ellison, N., Steinfield, C. y Lampe, C (2007): The Benefits of Facebook "Friends:" Social Capital and College Students' Use of Online Social Network Sites. Journal of Computer-Mediated Communication. Volume 12, Issue 4, pages 1143-1168, July 2007

Espuny, C. et al. (2011) Actitudes y expectativas del uso educativo de las redes sociales en los alumnos universitarios. Revista de Universidad y Sociedad del Conocimiento. Barcelona.

Ferrés, J. (2008). La educación como industria del deseo. Un nuevo estilo comunicativo. Barcelona: Gedisa.

Furth, H.G. (1992). El conocimiento como deseo. Un ensayo sobre Freud y Piaget. Madrid: Alianza, Col. Psicología.

Johnson, D. W., Johnson, R. (1989). Cooperation and Competitions. Theory and Research. Edina, MN. Interaction Book Company.

Slavin, R.E. (1987). Cooperative Learning: Student Teams. (2 $2^{\mathrm{a}}$ ed.) Wahington. DC:National Education Association, 1987.

Piscitelli, A. (2008). Nativos digitales. Contratexto [en linea], (16), pp. 43-56. ISSN 1025-9945. Recuperado de: http://fresno.ulima.edu.pe/sf/sf_bdfde.nsf/OtrosWeb/CONT16PISCITELLI/\$file/02contratexto16\%20PISCITELLI.pdf

Piscitelli, A., Adaine, I., y Binder, I. (2010). El Proyecto Facebook y la Posuniversidad. Sistemas operativos sociales y entornos abiertos de aprendizaje. Colección Fundación Telefónica. Barcelona: Ariel. 
Poza Luján, J.L., Cabrera, M., Calduch, A., y Teruel, L. (2015). ¿Es posible usar las redes sociales en la docencia universitaria? Conferencia: CINAIC 2015. III Congreso Internacional sobe Aprendizaje, Innovación y Competitividad, en Madrid. DOI: 10.13140/RG.2.1.4993.9607.

Prensky, M. (2014). No me molestes, mamá, jestoy aprendiendo! Madrid: Ediciones SM.

Rebollo M., Poza-Luján J.L., Cabrera Méndez M., Albors A. M., Teruel M. D., Calduch M.A., MartínEsparza M.E., Lloret Romero N., Despujol I., Díez Somavilla R. y Fernández L. (2011) Calidad en los procesos de enseñanza-aprendizaje mediante medios y redes sociales en educación. IV Jornada de Innovación docente. UPV.

Sharples, M; Adams A.; et al. (2014). Innovating Pedagogy 2014: Open University Innovation Report 3. Milton Keynes: The Open University. Institute of Educational Technology, United Kingdom: The Open University,

Recuperado de: http://www.openuniversity.edu/sites/www.openuniversity.edu/files/The_Open_University_Innovating Pedagogy_2014_0.pdf

Shirky C. (2012) Excedente Cognitivo. Creatividad y generosidad en la era conectada.- Bilbao: Ed. Deusto

Tourón, J., Santiago, R., y Díez, A. (2014). The Flipped Classroom: Cómo convertir la escuela en un espacio de aprendizaje. Barcelona: Editorial Grupo Océano. 